\title{
To Study Cost of Quality Elements for Designing and Development of Quality by Operation Project in English Biscuits Manufacturers Pvt Ltd
}

\author{
Mirza Amin ul Haq*
}

\begin{abstract}
The term cost of quality (COQ) explains how an organization can use its resources to the best in order to raise its product's quality or services and prevent poor quality resulting in any form of failure. This information help organization in determining possible saving that could be achieved on process improvements. Due to the current situation of world economic and market competition, organizations are working to reduce their supply chain cost. For this being an employee of quality assurance department, selected the project to work in the reduction of cost of quality specifically appraisal cost because currently, the company has very high (approx. 70\%) of its Quality budget associated with quality control activities thus decided to design and development of "Quality by Operation Project "to optimize the cost of quality. The goals of this project are to measure, the appraisal cost enhance the skill of the production workforce and highlight steps to optimize costs and processes. To create a better approach that makes an employee responsible for their quality control and manufacturing a defect-free product.
\end{abstract}

Keywords: Cost of Quality, Quality Control, Appraisal Cost, Quality by Operation

JEL Classification: G21, M14

\section{INTRODUCTION}

\section{Background of the study/project}

Now a day's quality is considered one of the crucial factors in achieving success among competitors and is highlighted as the central value for the customer. Continuous improvement programs not only discuss the need of meeting customer requirements but also help to carry out it at the least cost. Moreover, Due to rapid changes in work economic conditions and market competition in the FMCG sector, our organization (English Biscuits Manufacturers Pvt Ltd) has assigned a companywide strategic goal to reduce the cost of manufacturing, overhead, COGS, etc. To achieve this strategic goal of the organization, every department of the supply chain has started to work on a Cost Leadership approach with the slogan/theme as 'CEOs Challenge'. Being an employee of Quality Assurance, I have been selected to work for the reduction of Cost of Quality by designing and developing of 'Quality by Operation' project. Supply chain management and specifically Quality assurance is the key function of any business and plays a vital role in the success and failure of the entire organization, firm, or business. Therefore, we must know or identify the ways to strengthen or flourish our business and the factors related to it.

\footnotetext{
*Assistant Professor, Faculty of Business Administration Iqra University, Karachi, Pakistan. Email: mirzaaminulhaq@gmail.com (CorrespondingAuthor)
} 


\section{Cost of quality concept}

This concept was developed in the early fifties and now well-reputed companies are very much conscious about it. Being a part of the quality management team, I am interested in writing a thesis on this topic. It would be a great challenge and excitement for me to carry out such a study.

Cost of Quality is the combination of conformance and non-conformance costs as stated by (Juran,1974). According to Schiffauerova and Thomson (2006), cost applied on the prevention of poor quality (like a quality appraisal, inspection) is referred to as cost of conformance, and cost of non-conformance is the cost that occurred due to the product and service failure (for example, rework and returns). The concept of cost of poor quality is well defined by Juran as, "the sum of the total of all costs that would disappear or vanish if there were no quality problems" (Juran \& Bingham,1974). Crosby's model (Crosby, 1979) and P-A-F scheme of cost categories are similar and take a price of conformance and non-conformance combinedly as cost of quality.

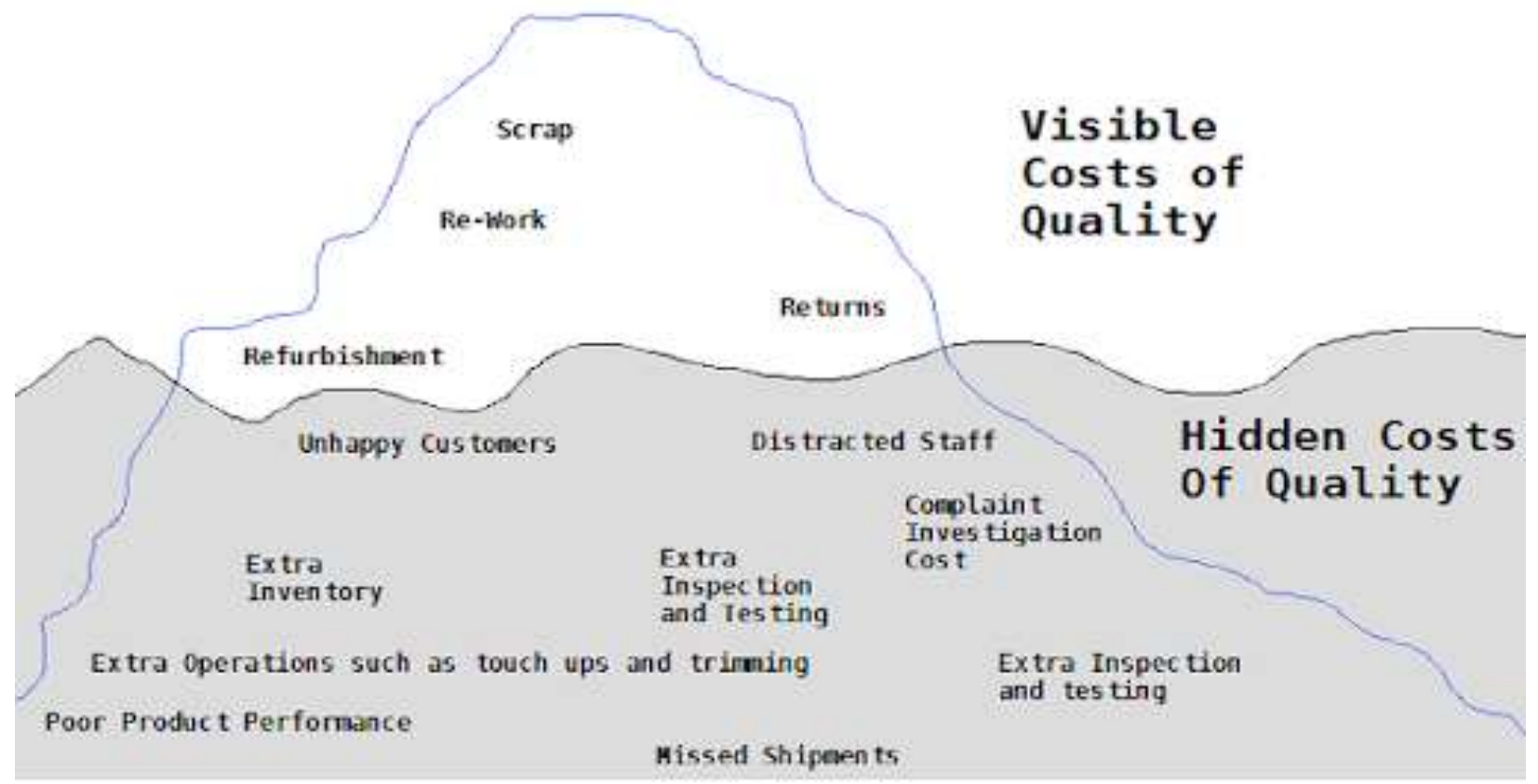

Figure 1: Crosby's Model

\section{APPROACHES USED FOR QUALITY COSTING Quality-costing approach}

It's a conventional approach in which prevention from bad quality, failure of any service and appraisal classifies the cost of quality. This approach includes the increase in prevention cost that helps in reduction of the cost of failure, also reducing the appraisal cost, eventually, the overall cost of quality is reduced.

\section{Quality-loss approach}

This approach uses the intangible and the tangible costs and losses, due to poor quality. The tangible losses are measured costs due to failure, and the intangible losses are the hidden failure cost. 
However, it only allows a rough idea of quality cost, but in case of lack of cost of data, this method could be the most feasible one.

\section{Process-cost approach}

This approach targets the cost for a process instead of a product or service. It could be helpful for the organizations that are mature enough to use TQM tools and whose tangible costs are relatively small. It needs to measure the cost of conformance in terms of the actual cost of product manufacturing for achieving certain standards, and the cost of non-conformance is associated with the failure of process execution up to the standard.

\section{Purpose of the research}

The purpose of this research is to critically analyze the different elements of the Cost of Quality and work to reduce appraisal cost (especially in-process quality inspection cost) by analyzing existing resources and proposing alternate solutions without compromising existing quality. Actions must be in compliance with company-defined SOPs and Specifications.

\section{Problem statement}

As already mentioned in the introduction, the CEOs challenge was the triggering point of the project. However, when we analyze the cost associated with the QA department, we found to come to know that the Total workforce (3P staff) ISIS 80 in the QA department in which Quality Inspectors, Lab Assistants, Lab Analyst and Sorters are included. Out of 80, 71 workers are in the Quality Control section who are designated in Mixing, Cutting, Oven and Packing Sections to check and control product and process quality. Company-operated 24 hours day and night and had 8 operation lines of Biscuits. Producing the best quality products is the key reason for the success of the organization that's why a high number of resources are dedicated to every section for ensuring good quality.

This is the reason that the maximum part of the budget is utilized in this section. Now that things are changing day by that's why management has decided to work on the cost of the quality project so that without compromising the product quality, we should re-design our process and establish any alternate solution which ultimately becomes the reason for quality cost reduction. Hence it is decided that " we would critically analyze the different elements of Cost of Quality and work to reduce appraisal cost (especially in-process quality inspection cost) by analyzing existing resource and propose an alternate solution without compromising existing quality. Actions must be in compliance with company defined SOPs and Specifications '. The project name would be decided as "' Quality by Operation".

\section{Scope of the project}

Quality Control section (In Process) will be the core section of this study because around 70\% departmental budget is associated with this section. Production skilled selected work force would also be the part of this project.

\section{Research question}

How would you optimize the cost of Quality (Appraisal Cost) without compromising product and process quality of Biscuits and cookies at EBM?

\section{Importance of the study}

The findings of the research will not be helpful for the Organization itself but also can be used as a reference study for onward working. The Company's strategic goal "CEOs Challenge " will be 
achieved by reducing the overall cost of Quality which was the ultimate goal. It will also be helpful for university students to understand the cost of quality in FMCG.

\section{LITERATURE REVIEW}

\section{Company profile}

EBM, the makers and the pioneers of Peek Freans Biscuits (Pied Piper), among the packed biscuit producers in Pakistan for over 50 years. EBM leads in the manufacturing of biscuits and cookies since 1967 till today with the consistent providing best quality of the product and has over $45 \%$ share of the branded biscuit in the market. Biscuits are produced unique Peek Freans Pied Piper umbrella brand with the slogan statement" The Legend leads ".

EBM has approx. 155,000 tons of annual production capacity which is the largest in Pakistan. The company has all required and recognized international certifications such as ISO 9001:2015 (QMS), FSSC ISO 22000:2015, ISO 14001:2004 (EMS), HACCP, and IIP (Investors in People) recognition. Also awarded the Environmental Excellence Awards consistently year after year.

\section{Industry application of COQ:}

During the study of different relevant survey reports and articles, it is understood that most companies are not adopted the cost of quality approach. Shah and Fitzroy 1998) concluded from a survey that the concept of COQ is not commonly accepted or used by organizations. Philip Crosby also has a similar opinion as he seems to be disappointed by the incorrect quality cost estimation. (Crosby, 1979). Most of the smaller firms often don't even have a budget to monitor costs relevant to quality. (Porter \& Rayner, 1992). Similarly, when I discussed working on this project even in my organization, most of my colleagues even didn't know the concept of COQ. This makes my aim stronger to do this project. We will discuss here how different companies adopted the different models of COQ.

In a review of Research, Andrea and Vince, analyze different COQ models and best practices used in different companies and concluded that Companies that have a quality system and CI programs but still didn't use COQ to cut down the cost of quality, and those who use it are found successful in reducing cost and improving overall quality (Schiffauerova \& Thomson, 2006). As we know quality is an integral part of the Supply Chain and introducing the COQ approach will ensure overall cost due to the probability of reduction of defects thus resulting in the reduction of additional cost due to corrective actions (Ramudhin et al., 2008)

A minor reduction in COQ will give significant profit to the company hence, the organization should consider COQ as an integral part of the improvement process and it targets the cost to achieve customer satisfaction. (Kiani \& Shadlen, 2009). The cost reduction improves quality and increases productivity. These cost reduction initiatives are achieved by defect reduction, eliminating waste by reworking on it, and by reducing machine idle time. (Harrington, 1987)

\section{Concept of COQ:}

As discussed earlier, the COQ is a combination and sum of conformance and the nonconformance quality cost. According to Crosby, it's not quality that costs an organization, instead, it's the cost of measures that are not taken properly at the first stage (Crosby, 1979). Feigenbaum (1951) 
has given a classification of cost, in association with conformity, on four different dimensions, discussing them below as:

\section{Prevention costs}

Prevention costs can be explained as the cost of activities and procedures done to prevent poor quality of product or service. E.g. Quality planning, Supplier evaluation, and Quality training and development, etc.

\section{Appraisal costs}

This includes the cost of measures taken to estimate, evaluate, and audit products or services, to achieve certain quality standards. E.g. Inspection and testing of materials, In-process Inspection, finished goods inspection, and quality audits, etc.

\section{Internal failure costs}

All the costs resulting due to failure in meeting the required standard or the failure to satisfy customer needs before the product delivery are included under the heading of internal failure costs. E.g. Rework, Delays, Re-designing Shortages Re-testing and Downtime, etc.

\section{External failure costs}

Counts all the cost that occurs due to the non-conformance of the product or service during or after the delivery to the customer. E.g. Complaints, Repairing goods and redoing services, Warranty, Customers' bad will and Losses due to sales reductions, etc.

\section{Generic COQ Models}

According to Schiffauerova quality cost can be divided into the following four categories:

1. P-A-F or Crosby Model

2. Opportunity cost models

3. Process cost models and

4. ABC (activity-based costing) models (Schiffauerova \& Thomson, 2006)

The most common among these models is the P-A-F model, i.e. the Prevention-AppraisalFailure approach (Schiffauerova \& Thomson, 2006) and it's the basis of most existing COQ models (Sandoval-Chávez \& Beruvides, 1998).

-The first PAF (Prevention-Appraisal-Failure) model invests in appraisal and prevention activities that reduce the failure cost. It helps in cost detection specifically related to quality and is easily expressed in terms of percentage of the total cost (Porter \& Rayner, 1992)

-Crosby, 1979 defines the cost of quality as the sum of the price of conformance and the price of non-conformance.

- Another COQ model is based on opportunity loss and intangible cost. Intangible costs measure all the costs that occurred as a result of customer loss and also due to the decrease in revenue, as a result of failure in assuring conformance. Opportunity cost can be well explained under three components: underutilization of installed capacity, inadequate material handling, and poor delivery of service. (Sandoval-Chavez \& Beruvides, 1998)

- The process cost model given by Ross (1977) and first used for quality costing by Marsh (1989). This approach focuses on the process instead of the product. This model explains the cost of conformance as the cost of the process for producing a standard product or service 
for the first time, whereas, the cost of non-conformance is explained as the cost applied to the process which failed to meet the required standards. Both of these can be measured at any point in the process. Porter and Rayner (1992) figured out if there is further need of expenditure for taking preventive actions or a redesign needed. This model is also used in Total Quality Management (TQM) recognizes a much better and integrated approach to quality than a P-A-F model (Porter \& Rayner, 1992).

- An activity-based costing (ABC) model was given by Cooper and Kaplan (Cooper, 1988; Cooper \& Kaplan, 1988). ABC traces out the cost of resources and the cost of activities of the respective process to cost the objects. It's an approach to identify the quality cost of the product rather than a COQ model, and it helps in effective quality cost management (Schiffauerova \&Thomson, 2006).

We will adopt different approaches for the project defined above but the project is most more relevant to Process Cost Model Approach, however will be using different best practices as well for reducing COQ at optimum level without compromising product and process quality specified by the company. Sailaja et al. (2015) was also used the same Process Cost model approach was also used by to identify the direct quality cost elements and hidden quality cost elements. Prior studies have shown that the hidden cost of quality is greater than thrice than that of the direct quality cost in an organization. But proper tracking and root cause analysis can help reduce or eliminate hidden costs. (Sailaja et al., 2015)

The P-A-F model is the only recognized model as an international standard for quality cost calculation. However, it categorizes the cost in a schematic manner and also has many constraints that's why it fails in a TQM program, which requires a process approach. (Porter \& Rayner, 1992). They also mentioned a few steps for adopting the process cost Model in TQM implementation:

- Design process flowchart and mark process owners.

- Highlight critical stages of the process.

- Estimate cost of Quality at different stages.

- Classification of costs into materials, equipment methods or human resources etc.

- Priorities failure costs and work to reduce non-conformance cost by selecting critical process steps.

- On a regular basis monitor and review conformance and nonconformance costs for further improvements and Changes.

Sandoval-Chávez and Beruvides (1998) also adopted P-A-F and Process cost model to estimate process cost in the continuous process industry (Sandoval-Chávez \& Beruvides, 1998).

This model talks about a significant part of the cost but hidden and opportunity costs are left uncovered. Even in the companies that have cut down the quality cost successfully, they still don't consider manufacturing and design losses which can provide opportunities to improve the system and decrease investments (Giakatis et al., 2001).

\section{Benefits of COQ:}

COQ has many advantages to the organization as discussed below: 
- The information is gathered in a systematic way and can be served as a baseline due to which improvement can be observed.

- COQ system can serve as a valuable input in a long-term perspective. It can help in designing process plans, giving information on how to analyze processes in order to improve quality expenditures. (Campanella \& Weemees, 1990; Greising, 1994).

- It helps to identify time lags in quality pay-offs, helps to relate between cost categories, and identifies shortcomings of the system (Campanella \& Weemees, 1990).

- This approach can provide managers with a tool of managerial techniques and procedures.

- This system should be planned properly and combined with CI efforts, to help an organization in generating valuable information related to quality. (Czuchry et al., 1995)

- COQ program aware of quality issues and identify main areas of improvement, thus helping to take preventive measures and improve control systems.

- COQ is very useful at organizational levels as it motivates top management giving them profit opportunities, by applying and maintaining a quality culture, continuous improvement (Howard, 2001).

\section{METHODOLOGY}

\section{Research Methodology}

The main objective of this research project is to identify and analyze the cost of quality (appraisal cost) involve in executing Quality Control Process activities at English Biscuits Manufacturers Pvt Ltd so that the project " Quality by Operation " can be designed and developed to achieve CEO's challenge (described in introduction chapter). The work should be according to scientific techniques. Therefore, initially, there would be a need to identify all those elements and process steps that are included in this study. There would be some parameters that are Quantitative and some are qualitative.

\section{Methodology adapted for this study}

- Design Process Flow along with the activities related to the quality control section

- Design and develop workforce (labor) strength and their initial Skill Matrix

- Design and develop skill development and training program plan

- Analyze elements of Quality Control Budget Specifically Revox

- Analyze skill matrix before and after project implementation

- Comparative analysis of internal and external complaints

At least one year of data would be studied for comparative analysis after implementing the Quality by Operation Project.

\section{Target Population:}

The Target population would be the Quality Control Section and production department only skilled workforce of Company English Biscuits Manufacturers Pvt Ltd.

\section{Sampling Criteria}

- Employee must be more than 1-2 years working experience in EBM

- Employee must be held at least intermediate education degree 
- Only skilled workers such as (Line Supervisors, Coordinators, Operators) would be considered for skill development.

- Internal quality complaints and external quality customer complaints data would be taken (as secondary data) for comparative analysis and to gauge project effectiveness

- Only Quality Inspectors deputed for online inspection would be considered. (Lab assistants and online sorters are excluded)

\section{Sample Size}

Only those workforces would be selected that could qualify in the initial skill assessment and fulfilling in sampling criteria mentioned above.

\section{Technique of Sampling}

Probability Sampling Techniques would be used for this study because population size in known. We are studying within a company data comparing them with general theories shared by a different researcher in their articles.

\section{Collection of data}

Data which is first-time data collected directly by the researcher called "Primary Data" (Mesly, 2015) for skill matrix before and after the project "Primary Data "would be collected from selected workers. Secondary Data is the data already collected by others. (Mesly, 2015) Source of Secondary is EBM SAP system for internal and external quality complaints to check the effectiveness of the project" Quality by Operation "

- For skill matrix, the first initial assessment would be made before the project starts with a selected and defined workforce of production i.e. Supervisors, Line coordinators, and Operators.

- Dimensions/area of assessment would be divided into a). Health and Safety, b) Good Manufacturing Practices (GMP) guidelines, c) Process and Product quality standards, and d). CRQS product defects manual.

- Questionnaire would be made in which at least 10 questions would be included from each area mentioned in the above point.

- The same data would be collected after implementing "Quality by Operation" project to know the skill level of the production team.

- To check the effectiveness of the project at least three years past data of internal and external complaints would be collected from the SAP system.

- Internal and external quality complaints data would be evaluated after project implementation.

\section{Research Approach}

The Deductive Research Approach would be adapted for this study because it starts with the statement or hypothesis and is then tested to check is true or not through observations. It moves from general to specific, so we take general theories shared by different researchers and apply them in our company to check the results. Data and observations would be evaluated and then proposed techniques would be implemented to test the hypothesis. 


\section{DATA ANALYSIS AND DISCUSSION}

This is a type of case study implemented this year in EBM. As we have discussed in the methodology chapter, how we adopted and make the production team accountable for quality inspection online, instead of quality inspectors. In this chapter, we will show outcomes of the system implemented through worker skill matrix, internal and external quality complaints.

\section{Selection of Skilled Work Force}

The plan was to utilize trained and accountable production line supervisors and coordinators to perform quality inspections and make them responsible for the online decision. For this purpose, we initially selected the resources who were fulfilling the sampling criteria prior to skill and knowledge assessment being taken. The written and oral interview/assessment of workforce were taken which contained questions from the following topics. 10 questions from each topic were asked and marked accordingly.

Table 2: Description of Interviewees/Assessments

\begin{tabular}{cccccc}
\hline S. No. & Position & Total Experience & Tenure & Gender & Age \\
\hline 1 & Line Supervisor & 15 years & 9 years & Male & 42 \\
2 & Senior Operator & 11 years & 8 years & Male & 39 \\
3 & Trainee Operator & 4 years & 3 years & Male & 35 \\
4 & Line Co-Ordinator & 6 years & 3 years & Male & 33 \\
\hline
\end{tabular}

Topics were:

1. Environmental and Health Safety (EHS)

2. Good Manufacturing Practice (GMP)

3. Sooper Product/material specification

4. Jam Delight Product/material specification

5. Choco DC/Vanilla Product/material specification

6. Sooper Process standards

7. Jam Delight Process standards

8. Choco DC/Vanilla Process standards

9. Defect awareness

10. Decision making on non-compliance

\section{Environmental and Health Safety (EHS)}

Responses of the interviewees

"According to our stats of interviews, environmental and health safety awareness is very much improved in the last couple of years due to induction of EHS department"

"Environmental and health safety awareness is very much important in any manufacturing industry that why we focused on"

"We believe safety is first which is our core part of company culture."

"Our company focused on EHS policy because the company cares for our employees." 


\section{Good Manufacturing Practice (GMP)}

Responses of the interviewees

"EBM is always followed Good manufacturing practices and regular training is also provided by management."

"GMP is very essential for any food industry and Alhamdulillah EBM is following the world's best practices."

"I have seen continual improvement in GMP from last 5 years. EBM encourages continual improvement."

"We must follow GMP and have good care of our hygiene while handling food products. '

\section{Product and Process Standard}

\section{Responses of the interviewees}

"EBM has its own product standards that complied strictly by the QA department. "

"Quality of the product can never compromise."

"We always keep product specifications online to run smooth production."

"Sooper is very high market share and known due to its quality."

"EBM has good quality control in compliance of product quality and Jam delight is one of the good brands in the market"

"We never compromise on quality and keep quality consistent. "

"Jam Delight's Jam has natural taste and aroma and we love this product"

"Jam delight defects are very different from other products due to its jam application of top of the biscuit."

"Choco double chocolate has good feedback in the market and it's we always try to produce good quality products."

"We must follow the standard while running product online and any sub-standard product always discarded."

"Process standard is very important to comply and we always set process parameters first before running any product. "

"Cut per minutes, Oven Profile is a very important parameter while producing biscuits and we always check time to time."

"We always report timely for any deviation that occurred during online production."

"Sooper is very high market share and known due to its quality."

\section{Process Flow Design}

The process flow consisted of eight biscuit operation lines that passed through four different sections those were: mixing section, where 3 inspectors were allocated for the inspection. Then the product reached the cutting section, with four-line inspectors. After cutting the product accelerated to the oven and then the packing section, where 4 and 8 inspectors were assigned duties. Section-wise product flow, number of quality inspectors, and assigned duties of the inspector are summarized in the following flow chart. 


\section{Mixing Section}

- 3 Inspectors for 8 lines

- To Inspect mixing ingrediants and area hyegine, sugar and sifter area

\section{Cutting Section}

- 4 Inspeotors 8 lines

- To Inspect of wet weight, Metal Detector, area GMP complaince and $\mathrm{CPM}$

\section{Oven Section}

- 4 Inspetors for 8 lines

- To Inspect dry weght product sensory, oven profile and area GMP compliance
Packing Section

- 8 inspetors for 8 lines

- To inspect CRQS parameters, CCP verification, batch code primary, secondary an tertiary packaging, area GMP

Figure 2: Four different Section

Inspectors in the mixing section were assigned to weigh raw ingredients according to the recipe, check cleanliness and hygiene to ensure GMP. They were also assigned to ensure the same in the flour sifting area.

\section{Mixing Section}

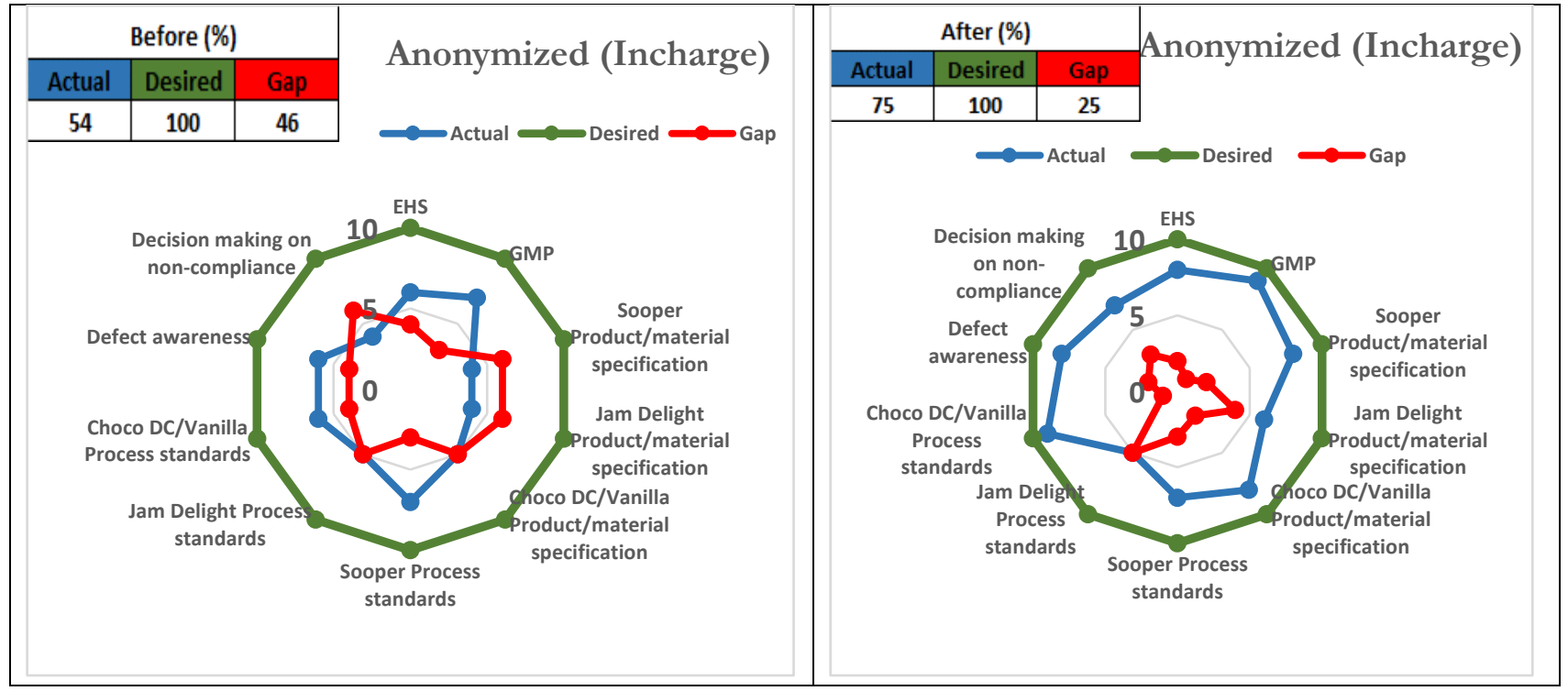




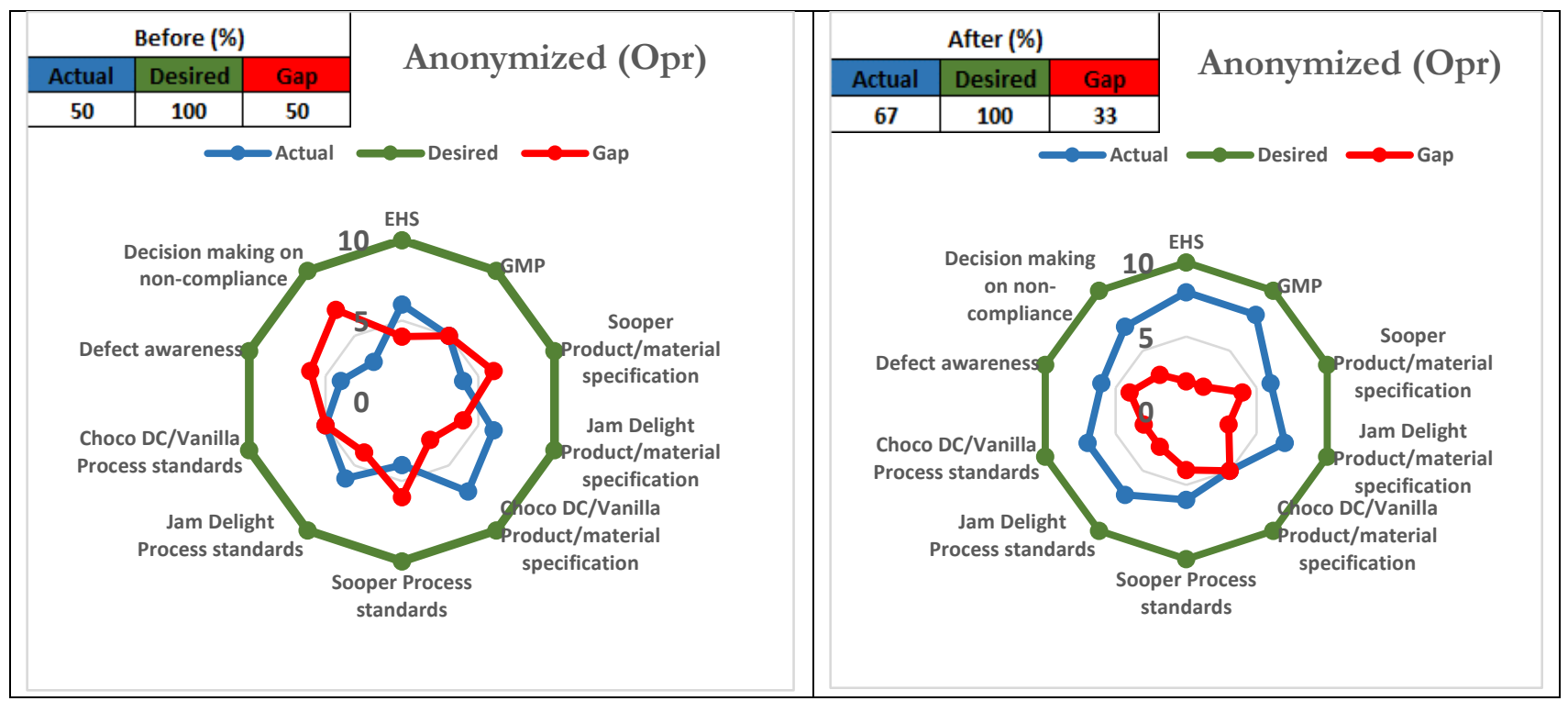

In the cutting section inspectors were instructed to check the wet weight of the biscuit, check metal detectors that were installed above the conveyer belt on which the biscuit was passing. The inspector assure the metal detector's working by standard strips. Cleanliness and hygiene conditions were monitored by the inspector in the given area and the cuts per minute of biscuits were also checked.

\section{Cutting Section}

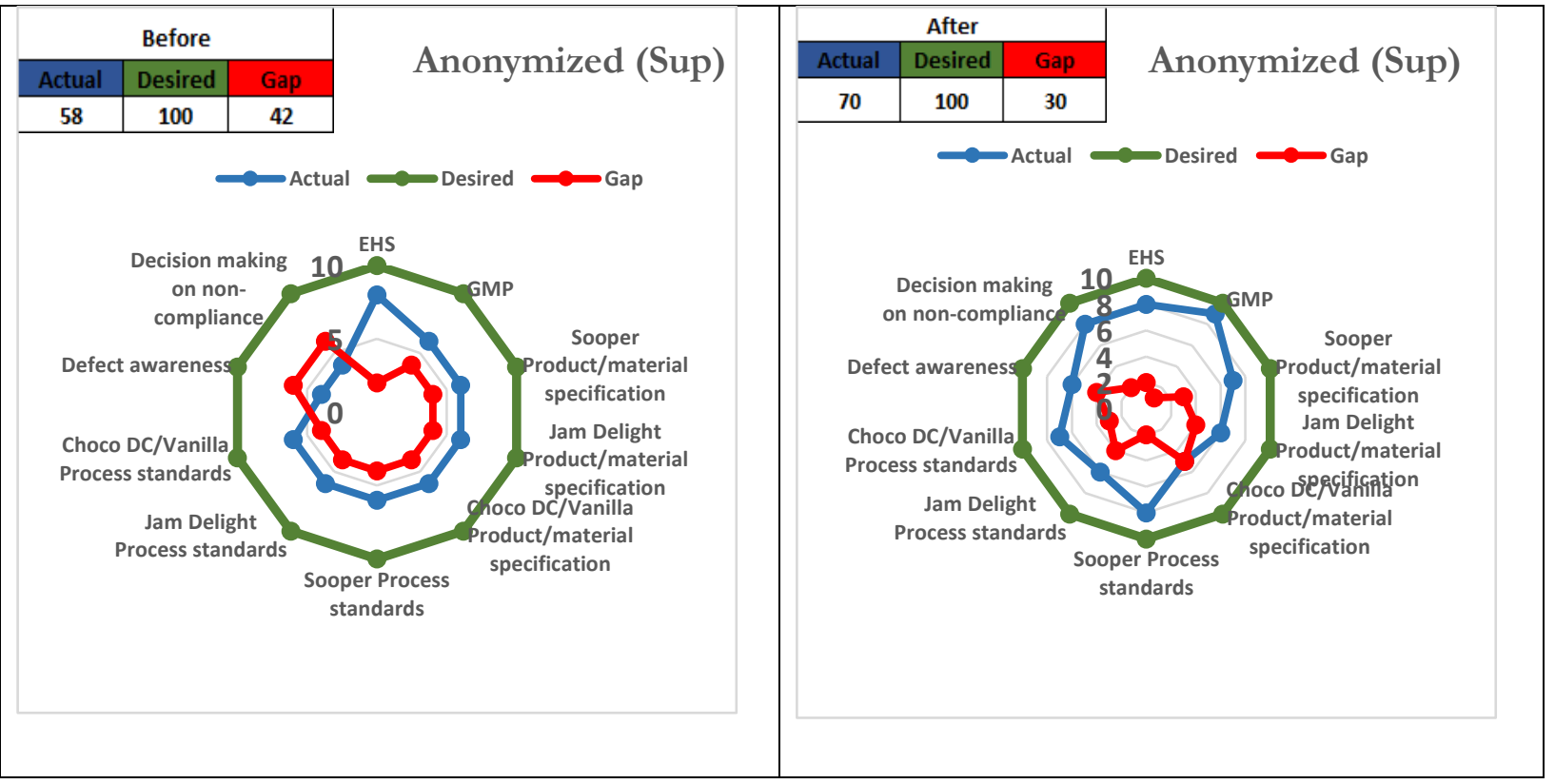




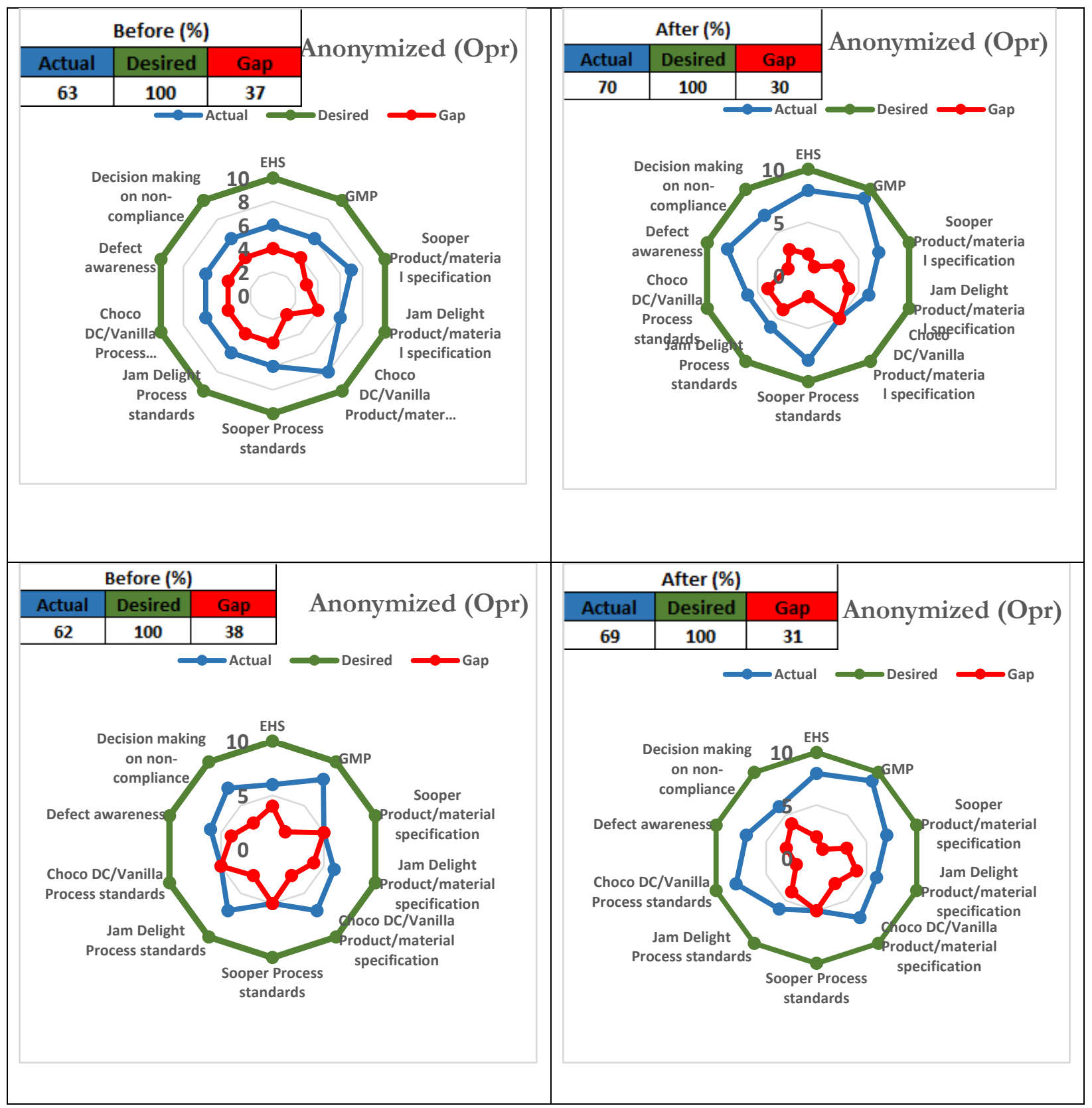

In the oven section inspectors checked for dry weight, conducted sensory of the biscuits and the GMP compliance. Inspectors in packing were checking CRQS parameters including packed weight, level one packaging defects, level two product defects, primary, secondary, and tertiary coding defects, coding verification machine speed, area hygiene, and GMP compliance.

Skill Matrices of Production Workers

Before the plan implementation skills of inspectors were gauged. Then they were trained for a month, to make them aware of defects and enhance their skills. They were trained for decisionmaking on non-compliance, defect awareness, different biscuits process standards, GMP, etc. After training, the inspectors were projected on pilot lines and engaged in practicing and onsite learning. 
This activity continued for one month. Then the inspectors were assigned duties on lines and were made accountable for that.

After three months, inspectors were tested for their skills as done previously before training, and data was collected. Their skills were seemed to be improved.

\section{Oven Section}

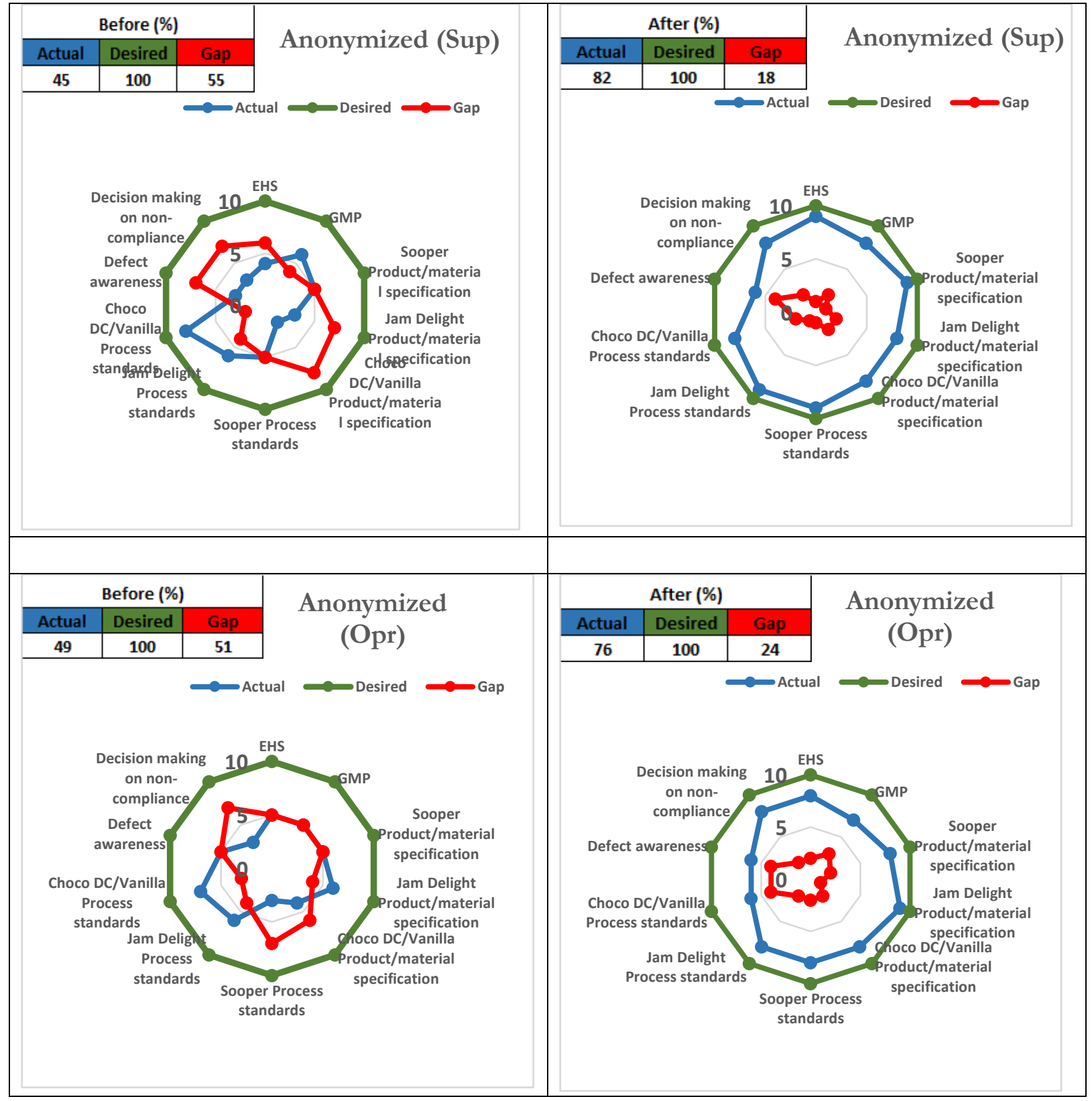




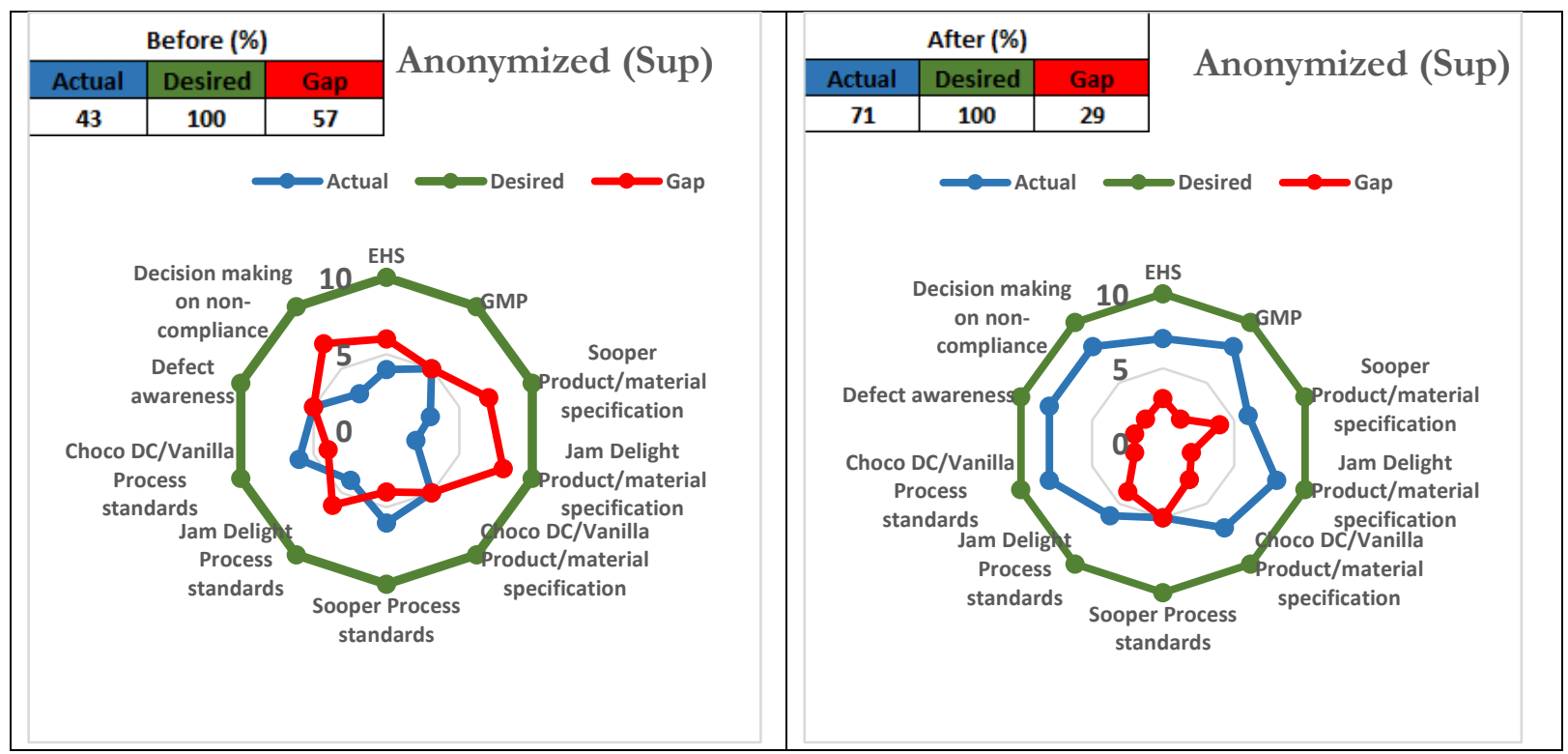

- Initially the project was started only on a single line i.e Line \# 3 and after one month, replicated to all other 8 lines.

- Quality inspection frequency in the mixing section was 4 times/12 hrs. Each line was inspected then assigned to the mixing operator and supervisor to check as guided. The Quality team only conduct audit or verification randomly 2-3 times per shift

- Quality inspection frequency in the cutting section was $1 / 2$ an hour, to check wet weight, Metal Detector, CPM, and GMP Condition. It was then shifted to the cutting operator to perform the same and record in the datasheet.

- Quality inspection frequency was also $1 / 2$ an hour. Parameters checked were dry weight, oven profile, product sensory attributes, which were then shifted to the oven line operator to perform the same and record data in the sheet.

\section{Internal Quality Complaints}

As the plan was implemented, the production team became more accountable for the quality. Their focus was increased and showed more responsibility against quality complaints. As a result, internal complaints showed a decline over time.

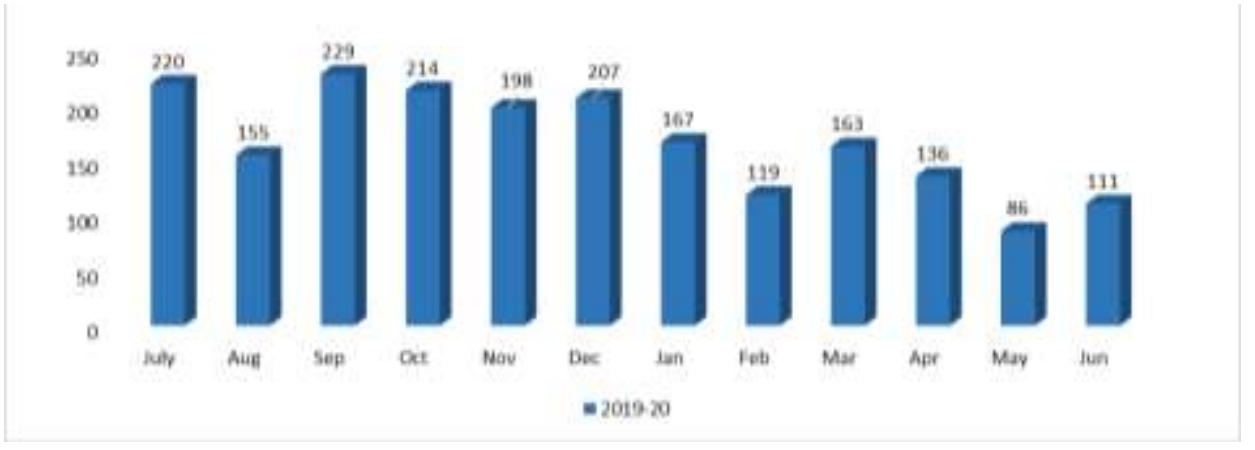

Figure 3: Internal Quality Complaint 


\section{Customer Complaint Trend}

Training the production workers and making them accountable showed no significant results in the context of external complaints. The reason for such a response is customer complaint culture. Customers are not much aware or have not developed a culture of making complaints. Secondly, the company was already working on very low customer complaints, that's why the change in customer complaints cannot be correctly obtained. Another reason for obtaining customer complaints trend is the product that is dealt by the company. Biscuit is a product in which the rate of complaints is already low. The complaints trend of the current year and previous two years is shown below.

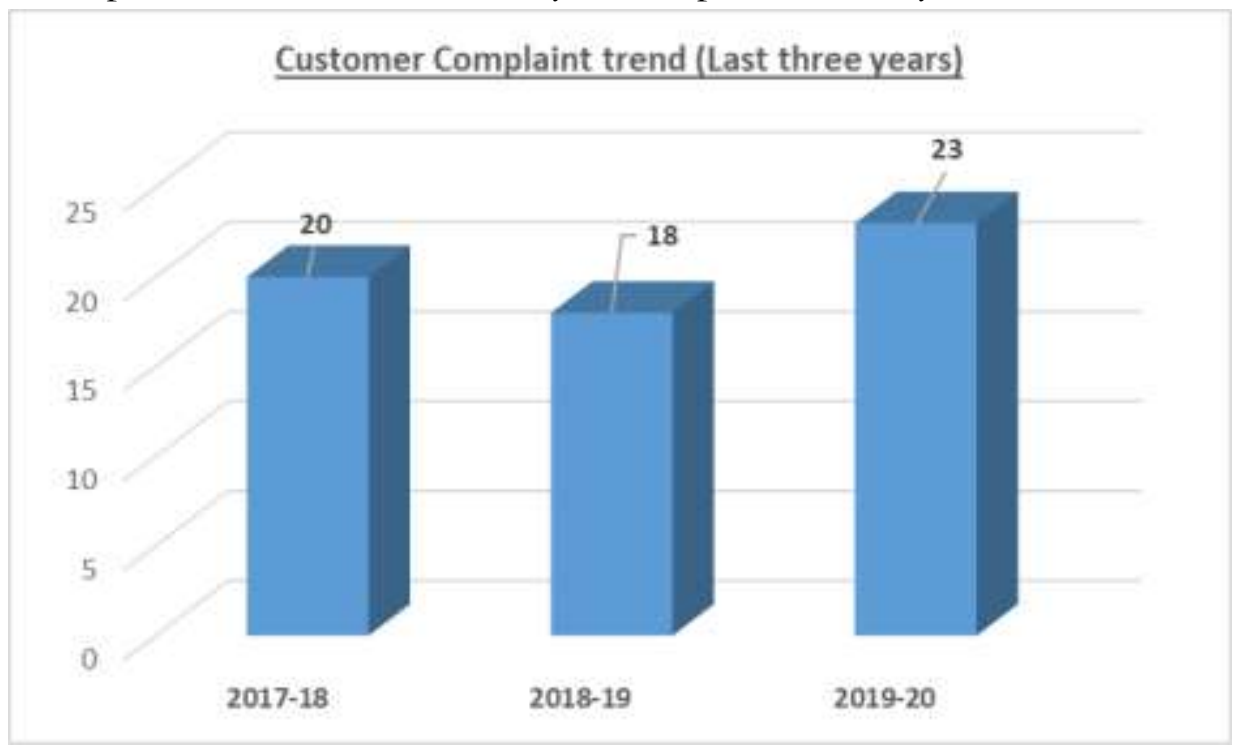

Figure 4: Customer Complaint

\section{CONCLUSION AND RECOMMENDATION}

\section{Conclusion}

The project was conducted to study the quality cost elements. The production team workers were trained to check for non-compliance and work as a quality inspector. According to the hypothesis, a Quality by operation project was found successful. The project increased production resources. Previously, there was a single inspector from the quality department to check and ensure quality now that figure increased by the number of workforces that were trained in all different sections. And thus, production's multiple resources developed.

As the production workers were skilled enough to respond to quality complaints and ensure quality, a quality culture developed throughout the operation. Quality assurance became a duty and culture of all, instead of being the sole duty of the quality department.

With the development of quality awareness in the production department, the need for quality inspectors in the quality department was decreased. So, the quality control was rationalized and its eight resources were reduced. This resource reduction eventually resulted in appraisal cost reduction and around there was a saving of 4.2 million Rs per annum. 


\section{Challenge and Limitation}

One of the challenges faced in this project was workers turnover. As the hiring firing of production workers is usually frequent than skilled quality control inspectors. This challenge was met by fixing that workforce throughout the project.

This could also be a limitation for others in implementing it to a company. i.e., making production workers accountable to ensure quality. So, the company should have to fix some skilled workforce in order to achieve the said results.

\section{REFERENCES}

Campanella, R. G., \& Weemees, I. (1990). Development and use of an electrical resistivity cone for groundwater contamination studies. Canadian Geotechnical Journal, 27(5), 557-567.

Cooper, H. M. (1988). Organizing knowledge syntheses: A taxonomy of literature reviews. Knowledge in society, 1(1), 104-126.

Cooper, R., \& Kaplan, R. S. (1988). How cost accounting distorts product costs. Strategic Finance, 69(10), 20.

Crosby. P.B, (1979). Quality without tears. McGraw-Hill.

Czuchry, M., Dansereau, D. F., Dees, S. M., \& Simpson, D. D. (1995). The use of node-link mapping in drug abuse counseling: The role of attentional factors. Journal of Psychoactive Drugs, 27(2), 161166.

Sandoval-Chávez, D. A., \& Beruvides, M. G. (1998). Using opportunity costs to determine the cost of quality: A case study in a continuous-process industry. The Engineering Economist, 43(2), 107124.

Feigenbaum, A. V. (1951). Quality control: Principles, practice, and administration: An industrial management tool for improving product quality and design and for reducing operating costs and losses. McGraw-Hill.

Giakatis, G., Enkawa, T., \& Washitani, K. (2001). Hidden quality costs and the distinction between quality cost and quality loss. Total Quality Management, 12(2), 179-190.

Greising, D. (1994). Quality: How to make it pay. Business Week, 8(8), 54-59.

Harrington, H. J. (1987). Poor-quality cost. CRC Press.

Howard, J. (2001). Mechanics of motor proteins and the cytoskeleton. Sinauer Associates Inc, Sunderland, USA.

Juran, J. M., \& Bingham, R. S. (1974). Service industries. Quality Control Handbook, McGraw-Hill, New York, USA.

Kiani, R., \& Shadlen, M. N. (2009). Representation of confidence associated with a decision by neurons in the parietal cortex. Science, 324(5928), 759-764.

Marsh, H. W. (1989). Age and sex effects in multiple dimensions of self-concept: Preadolescence to early adulthood. Journal of Educational Psychology, 81(3), 417.

Mesly, O. (2015). Creating models in psychological research. Springer.

Porter, L. J., \& Rayner, P. (1992). Quality costing for total quality management. International journal of production economics, 27(1), 69-81.

Ramudhin, A., Alzaman, C., \& Bulgak, A. A. (2008). Incorporating the cost of quality in supply chain design. Journal of quality in maintenance engineering, 14(01), 71-86 
Ross, S. A. (1977). The determination of financial structure: the incentive-signaling approach. The bell journal of economics, 8(1), 23-40.

Schiffauerova, A. \& Thomson, V. (2006). A review of research on the cost of quality models and best practices, International Journal of Quality and Reliability Management, 23(6), 647-669.

Sailaja, A., Basak, P. C., \& Viswanadhan, K. G. (2015). Hidden costs of quality: Measurement \& analysis. International Journal of Managing V alue and Supply Chains, 6(2), 13-25.

Sandoval-Chávez \& Beruvides, (1998). Using opportunity costs to determine the cost of quality: A case study in a continuous-process industry. The Engineering Economist, 43(2), 107-124. 